\title{
Hypercaptation à l'IRM pondérée en diffusion avec suppression des signaux corporels non pertinents
}

\author{
Fumio Shimada MD PhD, Miwa Misawa MD, Tomio Suzuki MD PhD
}

- Citation : CMAJ 2021 January 11;193:E48. doi : 10.1503/cmaj.191188-f

Voir la version anglaise de l'article ici : www.cmaj.ca/lookup/doi/10.1503/cmaj.191188

$\mathbf{U}$ ne femme de 78 ans a consulté au service des urgences pour une douleur fémorale droite présente depuis 2 semaines. Les résultats d'imagerie par résonance magnétique (IRM) laissant soupçonner des tumeurs osseuses, la patiente a été dirigée vers notre hôpital. La douleur, qui empirait en position debout et à la marche, s'est étendue à l'ensemble du corps 4 semaines après son apparition. Comme nous soupçonnions la présence de tumeurs osseuses métastatiques, nous avons réalisé une IRM corporelle pondérée en diffusion avec suppression des signaux non pertinents (DWIBS), qui a mis en évidence des zones chaudes diffuses s'apparentant à une hypercaptation dans de nombreux os. L'hypercaptation est un phénomène caractérisé par la fixation diffuse et symétrique accrue de radionucléides dans les os, mais pas dans les reins et la vessie, à la scintigraphie osseuse avec technétium $99 \mathrm{~m}$ et bisphosphonate. Elle peut révéler la présence d'une atteinte osseuse métastatique, d'une maladie osseuse métabolique ou d'une atteinte osseuse primaire répandue ${ }^{1}$ (figure $1 \mathrm{~A}$ ).

Durant son séjour à l'hôpital, la patiente avait un taux sérique de phosphatase alcaline normal (311 IU/L [plage de référence 106-322 IU/L]) et un taux de calcium presque normal $(10,8 \mathrm{mg} / \mathrm{dL}$ [plage de référence $8,4-10,1 \mathrm{mg} / \mathrm{dL}$ ]). Nous avons donc éliminé la possibilité d'une maladie ostéolytique diffuse et avons conclu que la patiente présentait plutôt un trouble de la moelle osseuse attribuable à des métastases venant d'une tumeur maligne inconnue. Nous avons alors réalisé une tomographie par émission de positrons au 18F-fluorodésoxyglucose
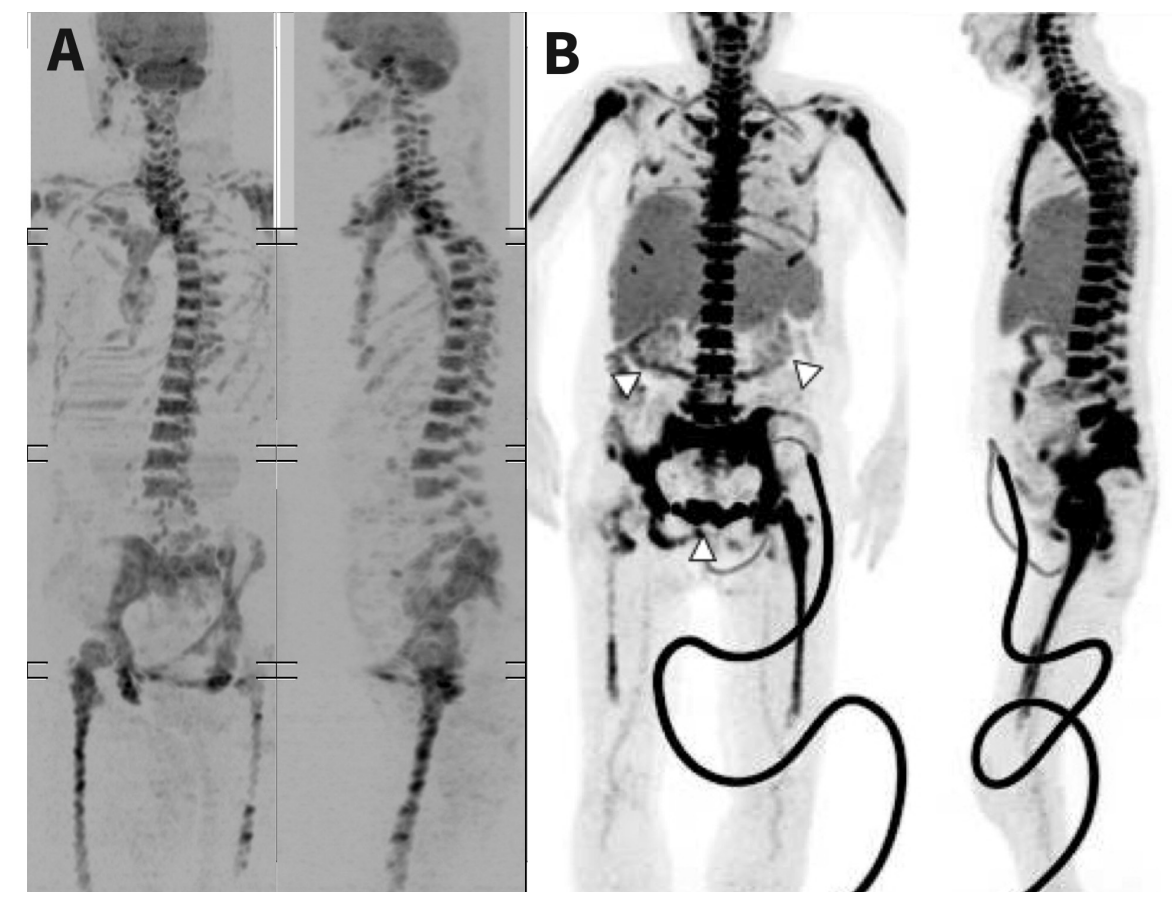

Figure 1 : A) Image par résonance magnétique (IRM) corporelle pondérée en diffusion avec suppression des signaux non pertinents (DWIBS; noir et blanc inversé) chez une femme de 78 ans atteinte d'un lymphome primaire à cellules B touchant la moelle osseuse. On observe des zones chaudes (en noir) à la mandibule, au corps vertébral, à la clavicule, au sternum, à la tête de l'humérus, au pelvis, au fémur et aux côtes. B) Tomographie par émission de positrons au 18F-fluorodésoxyglucose combinée à une tomodensitométrie (TEP-TDM) montrant une captation accrue des radionucléides à la mandibule, au corps vertébral, à la clavicule, au sternum, à l'humérus, à une partie des côtes, au pelvis et au fémur. Une hépatosplénomégalie est également visible, ainsi qu'une captation réduite des radionucléides aux reins et à la vessie (flèches blanches). La ligne courbe artificielle au bas du corps correspond à une sonde à demeure. 
La DWIBS est une méthode mise au point pour détecter les tumeurs à divers endroits du corps au moyen d'une IRM pondérée en diffusion ${ }^{2}$. On a démontré qu'elle est associée à un taux de détection des métastases osseuses équivalent ou supérieur à celui de la scintigraphie osseuse ${ }^{3}$. La DWIBS prend la moitié du temps de la TEP-TDM, et coûte le sixième du prix de cet examen (au Japon). Elle est particulièrement indiquée chez les personnes qui souhaitent éviter la radiation, de même que chez les femmes enceintes ou qui allaitent.

\section{Références}

1. Osmond JD III, Pendergrass HP, Potsaid MS. Accuracy of 99mTC-diphosphonate bone scans and roentgenograms in the detection of prostate, breast, and lung carcinoma metastases. Am J Roentgenol Radium Ther Nucl Med 1975;125:972-7.

2. Takahara T, Imai Y, Yamashita T, et al. Diffusion weighted whole body imaging with background body signal suppression (DWIBS): technical improvement using free breathing, STIR and high-resolution 3D display. Radiat Med 2004;22:275-82.

3. Stecco A, Lombardi M, Leva L, et al. Diagnostic accuracy and agreement between whole-body diffusion MRI and bone scintigraphy in detecting bone metastases. Radiol Med (Torino) 2013;118:465-75.
Intérêts concurrents : Aucun déclaré.

Cet article a été révisé par des pairs.

Les auteurs ont obtenu le consentement de la patiente.

Affiliation : Département de médecine générale, Osaka Medical College Hospital, Takatsuki, Osaka, Japon.

Financement : Cette étude a été financée par le département de médecine générale de l'Osaka Medical College Hospital.

Propriété intellectuelle du contenu : Il s'agit d'un article en libre accès distribué conformément aux modalités de la licence Creative Commons Attribution (CC BY-NC-ND 4.0), qui permet l'utilisation, la diffusion et la reproduction dans tout médium à la condition que la publication originale soit adéquatement citée, que l'utilisation se fasse à des fins non commerciales (c.-à-d., recherche ou éducation) et qu'aucune modification ni adaptation n'y soit apportée. Voir : https://creativecommons.org/licenses/by-nc -nd/4.0/deed.fr.

Correspondance : Fumio Shimada, gmd012@osaka-med.ac.jp 